\title{
Identification and genotyping of the etiological agent of tuberculous lymphadenitis in Ethiopia
}

\author{
Demissew Beyene ${ }^{1,2}$, Indra Bergval ${ }^{3}$, Elena Hailu ${ }^{1}$, Senait Ashenafi ${ }^{4}$, Lawrence Yamuah $^{1}$, \\ Abraham Aseffa ${ }^{1}$, Harald G. Wiker ${ }^{5,6}$, Howard Engers ${ }^{1}$, Paul Klatser ${ }^{3}$, Lisbet Sviland ${ }^{7,8}$ \\ ${ }^{1}$ Armauer Hansen Research Institute (AHRI), Addis Ababa, Ethiopia \\ ${ }^{2}$ Centre for International Health, University of Bergen, Bergen, Norway \\ ${ }^{3}$ Royal Tropical Institute (KIT), KIT Biomedical Research, Amsterdam, The Netherlands \\ ${ }^{4}$ Department of Pathology, Medical Faculty, Addis Ababa University, Addis Ababa, Ethiopia \\ ${ }^{5}$ Section for Microbiology and Immunology, the Gade Institute, University of Bergen, Bergen, Norway \\ ${ }^{6}$ Department of Microbiology and Immunology, Haukeland University Hospital, Bergen, Norway \\ ${ }^{7}$ Department of Pathology, Haukeland University Hospital, Bergen, Norway \\ ${ }^{8}$ Section for Pathology, the Gade Institute, University of Bergen, Bergen, Norway
}

\begin{abstract}
Background: In Ethiopia, little has been done to assess how Mycobacterium bovis has contributed to human tuberculosis, though the population routinely consumes unpasteurized milk and raw meat. The aim of this study was to determine the proportion of $M$. tuberculosis and $M$. bovis as etiological agents of tuberculous lymphadenitis (TBLN).

Methods: Patients with lymphadenopathy $(\mathrm{n}=171)$ were included in a cross-sectional study at Butajira Hospital, Southern Ethiopia. Lymph node biopsies were cultured. Patients' HIV status was identified. DNA from positive cultures was tested by PCR to identify $M$. bovis and $M$. tuberculosis. Isolates were genotyped by multiplex ligation-dependent probe amplification (MLPA) assay.

Results: Among 171 patients, 156 had culture results. Of these, 107 (69\%) were positive for M. tuberculosis complex (MTC). Six of the 10 HIV-positive patients were culture positive. M. tuberculosis specific sequences were identified in the DNA of each of 100 samples as assessed by RD10 targeted PCR, and each of the 95 isolates exhibited the M. tuberculosis specific TbD1 deletion by MLPA analysis. No $M$. bovis was identified. These results indicate that all the isolates were modern M. tuberculosis strains. Furthermore, MLPA studies confirmed that $42 \%$ of the isolates showed the Haarlem genotype and $12 \%$ displayed sequences compatible with INH resistance. No mutations conferring resistance to ethambutol or rifampicin were detected.

Conclusions: Our data showed that M. tuberculosis strains had common characteristics with strains causing pulmonary TB, which appears to be the main etiological agent of TBLN.
\end{abstract}

Key words: etiological agent, tuberculous lymphadenitis, Ethiopia

J Infect Dev Ctries 2009; 3(6):412-419.

Received 26 March 2009 - Accepted 21 May 2009

Copyright ( 92009 Beyene et al. This is an open-access article distributed under the Creative Commons Attribution License, which permits unrestricted use, distribution, and reproduction in any medium, provided the original work is properly cited.

\section{Introduction}

Tuberculosis (TB) is one of the most serious public health challenges in Ethiopia. According to a World Health Organization (WHO) report in 2008 [1], Ethiopia is number seven among the most highly affected countries on the TB-burden list. Primarily considered a pulmonary disease, TB can affect almost any organs. According to the National TB and Leprosy Control Program (NTLC) of Ethiopia [2], pulmonary TB (PTB) accounts for $63.4 \%$ of all TB cases and extra-pulmonary tuberculosis (EPTB) accounts for $36.6 \%$ of TB cases. In Ethiopia, $80 \%$ of EPTB is tuberculous lymphadenitis (TBLN) [2].
HIV has been identified as one of the most important factors contributing to an increase in the incidence of tuberculosis, particularly in Africa [1]. Treatment of co-infected patients is complicated by toxicities secondary to drug-drug interactions. HIV infection also makes TB diagnosis difficult, as the infection leads to treatment delays and hence increased morbidity and mortality rates. Drugresistant TB is emerging as a significant problem as a result of inadequate treatment [1].

Among members of the Mycobacterium tuberculosis complex (MTC), M. tuberculosis and $M$. bovis have been implicated as the most common causes of tuberculous lymphadenitis (TBLN) 
[3,4,5,6]. In Ethiopia, little has been done to identify how $M$. bovis causes human TB, despite the population's routine consumption of unpasteurized milk and raw meat. A skin testing study from Central Ethiopia reported $13.5 \%$ as the average prevalence of bovine $\mathrm{TB}$ in cattle and $22 \%, 12 \%$, and $12 \%$ prevalence in Holstein, Zebu, and cross-bred cattle respectively [7]. Studies from Tanzania and Uganda report that $M$. bovis should be considered a pathogen of concern for people living in rural areas of Africa [8,9]. A study by Kidane et al. in 2002 [5] using DNA from fine needle aspirates (FNA) of TBLN patients from Butajira, southern Ethiopia reported that $17 \%$ of cases were caused by M. bovis and $83 \%$ by $M$. tuberculosis. However, previous studies report that the reduced yield of microorganism load compromises the interpretations of FNA in comparison to lymph node biopsies [10,11].

Molecular identification and characterization of MTC strains has seen several recent advances. Studies have shown that PCR-based assays are rapid, specific, and sensitive in differentiating species of MTC [12,13]. Parsons et al. in 2002 [12] reported that regions of differences (RD1 to RD16) variably deleted in other MTC members, but present in $M$. tuberculosis, could be used to differentiate species of MTC. However, PCR approaches performed directly on clinical material produces highly variable and inconsistent results [14]. Conversely, MLPA allows the simultaneous detection of multiple mutations in the genome in one assay by amplification of sequence-specific probes rather than target DNA [15]. Recently, MLPA has been adapted to characterize MTC members [16]. M. tuberculosis specificity is achieved by targeting $16 \mathrm{~S}$ rRNA and IS6110 sequences. MLPA targets the most prevalent and clinically relevant drug resistance mutations as well as several markers specific for each principal genotypic group (PGG). In this study, positive cultures from biopsy were used as sources of mycobacterial DNA to identify the proportion of $M$. bovis and $M$. tuberculosis as the etiological agents of TBLN by using RD10 targeted PCR. Furthermore, isolates were analyzed by MLPA to identify the genotype of the strains and any possible drug resistance patterns that are prevalent in the area.

In the current study, we have readdressed the issue of mycobacterial identity in TBLN patients in Ethiopia by using lymph node biopsies rather than FNA aspirates to improve mycobacterial recovery, and MLPA to improve detection and specificity. In addition, we have utilized MLPA assay to ascertain genotype and drug resistance profiles of $M$. tuberculosis isolates.

\section{Materials and Methods}

Study Area

Butajira, in the Meskenena Mareko district of Southern Ethiopia (135 km from Addis Ababa), has an estimated population of 331,832 and an estimated geographic area of 872.5 square kilometers [17].

\section{Study Participants}

Ethical approval was obtained from AHRI/ALERT Ethical Committee (Reg.No.001/05) and the National Ethical Review Committee (NERC), (RDHE/190-64/2005). The formula used to calculate the sample size based on the hypothesis was $\mathrm{n}=$ $\left(1.96 / \log _{\mathrm{e}} \mathrm{f}\right)^{2}\left\{\left[(\mathrm{R}+1) /\left(\mathrm{RP}_{2)}-2\right\}\right.\right.$, where $\mathrm{R}$ is the relative risk, $\mathrm{P}_{2}$ is the proportion of the control group,

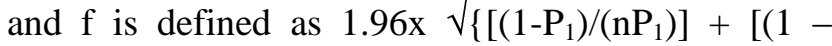
$\left.\left.\left.\mathrm{P}_{2}\right) /\left(\mathrm{nP}_{2}\right)\right]\right\}, \mathrm{n}$ is the number of subjects in each group and $\mathrm{P}_{1}$ is the proportion in group one. Patients with lymphadenopathy were screened using the national diagnostic algorithm for TBLN at Butajira hospital [18].

Patients who were clinically diagnosed with TBLN and signed the written consent form were included in our study. In total, 171 patients took part in the study, from October 2005 to July 2006. Patients who were critically ill, taking anti-TB drugs, had other forms of TB, were anemic, or had medical contra-indications or did not consent were excluded from the study. This study is part of a larger crosssectional study designed to develop serological diagnostic test(s) for TBLN.

\section{Collection of biopsy, FNA and blood specimens}

Excision biopsies and FNAs were taken from all patients. Biopsies were divided into two halves - one for histology and the other for culture. The culture biopsy was placed in a tube containing $1 \mathrm{ml}$ phosphate buffer saline (PBS) and the histology biopsy was fixed with $10 \%$ formalin. The FNA was used for Ziehl Neelsen (ZN) staining and PCR in some cases. Samples were stored at $4^{\circ} \mathrm{C}$ prior to and during transport to Armauer Hansen Research Institute (AHRI) for processing. The delay in transporting samples from our study site to the AHRI laboratory was not more than six hours.

\section{Culture}

All processing procedures were conducted using aseptic techniques in a class I safety cabinet. Biopsies 
were cut into pieces and decontaminated by $1 \%$ sodium hydroxide containing 3\% sodium dodecyl sulphate (SDS). The suspensions were shaken and the homogenates were centrifuged. The pellets were neutralized by $0.1 \%$ sulfuric acid containing $0.0008 \%$ bromocresol-purple. Finally, $100 \mu \mathrm{l}$ of each suspension was inoculated in two types of Løwenstein-Jensen (LJ) media, one containing glycerol and the other pyruvate to ensure the growth of $M$. bovis. The inoculated media were incubated at $37^{\circ} \mathrm{C}$ for up to eight weeks, while bacterial growth was observed weekly.

\section{HIV testing}

HIV testing was performed using the diagnostic algorithm assay kit, following the manufacturer's instructions and the National guidelines for HIV screening as described by Ethiopia's Ministry of Health,. The following three stages of the kit were used: Determine Lateral Flow test (ABBOTT Japan Co.Ltd, Mitato-KU, Tokyo, Japan, and AUST R7449); Capillus (Trinity Biotech PIV, Bray, Ireland); and Unigold (Trinity Biotech-PIC, Bray, Ireland). Patients who tested positive for any two of these tests were reported as positive. Appropriate positive and negative controls supplied by the manufacturers were considered at each stage of the HIV test.

\section{DNA extraction from frozen biopsy cultures}

DNA extraction was performed by the boiling method [19]. A loop-full of bacteria from positive cultures and reference strains of $M$. tuberculosis and $M$. bovis were transferred to a $1.5 \mathrm{ml}$ Eppendorf tube containing $400 \mu \mathrm{l}$ of 1xTE (Tris ethylene di-amine tetra acetic acid). The resuspended bacteria were heated for one hour at $80^{\circ} \mathrm{C}$ in a water bath and $2 \mu 1$ from the supernatant was used for PCR.

DNA extraction from $F N A$

DNA was extracted as reported by van Soolingen et al. in 1995 [20]. Briefly, a $400 \mu \mathrm{l}$ vial of FNA was incubated at $80^{\circ} \mathrm{C}$ for one hour to inactivate the bacteria. The bacteria were lysed using $10 \mathrm{mg} / \mathrm{ml}$ lysosyme (Sigma, Germany) and then digested with $10 \mathrm{mg} / \mathrm{ml}$ proteinase K (Sigma, Germany). DNA was extracted with chlorophorm-isoamyl alcohol (24:1) and precipitated with isopropanol. The DNA pellet was resuspended in $20 \mu \mathrm{l}$ of TE buffer and stored at $20^{\circ} \mathrm{C}$ until used for PCR.

\section{PCR amplification}

PCR targeting the RD10 region was conducted as reported by Parsons et al. 2002 [12]. The RD10 primers were RD forward (RD10F): 5 -CTG-CAACCA-TCC-GGT-ACA-C-3', RD internal (RD10Int): 5-GAA-GTC-GTA-ACT-CAC-CGG-GA-5 , RD reverse (RD10R): 5-AAG-CGC-TAC-ATC-GCCAAG-3`. The two primers (RD10F) and (RD10R) are complementary to the sequences flanking the deleted region and amplify a smaller PCR product (202bp) from strains where RD10 is deleted. The third primer (RD10I) is complementary to an internal sequence of RD10 and amplifies a product of different size (308 $\mathrm{bp)}$ when combined with one of the flanking primers (RD10F). The two flanking primers (RD10F and RD10R) are too far apart to efficiently amplify the entire region for the strain [12]. RD10 specific sequences are deleted not only in $M$. bovis, but in other MTC, such as M. caprae, M. microti, $M$. pinnipedii, and a subset of $M$. africanum. Moreover, the assay is not specific for $M$. tuberculosis because $M$. canettii and some $M$. africanum species are RD10 intact. Therefore, the RD10 PCR technique needs to be combined with the MLPA assay, which identifies the TbD1 region and is specifically deleted in modern M. tuberculosis isolates. The $25 \mu \mathrm{l}$ PCR mix (Hot start PCR master mix, Qiagen $\mathrm{GmbH}$ ) consisted of 2 $\mu 1$ of DNA template and $10 \mu \mathrm{M}$ of the two flanking primers and $50 \mu \mathrm{M}$ of the internal primer. The PCR program comprised of denaturation at $95^{\circ} \mathrm{C}$ for 15 minutes, then 35 cycles of denaturation at $94^{\circ} \mathrm{C}$ for one minute, annealing at $55^{\circ} \mathrm{C}$ for one minute and extension at $72^{\circ} \mathrm{C}$ for one minute, and a final extension at $72^{\circ} \mathrm{C}$ for 10 minutes in a thermocycler (Thermo-Hybaid, UK).

\section{Analysis of PCR product}

Ten $\mu 1$ of the amplified products and $2 \mu 1$ of $6 \mathrm{x}$ PCR loading dye were analyzed on a $1.8 \%$ agarose gel containing ethidium bromide. Electrophoresis was performed at $100 \mathrm{~V}$ for one hour. One hundred bp DNA molecular weight markers were used. Amplification of a $308 \mathrm{bp}$ PCR product indicates $M$. tuberculosis whereas amplification of a $202 \mathrm{bp}$ product indicates $M$. bovis. However, further confirmation of MLPA through the absence and presence of TbD1 region is needed.

$M L P A$

MLPA analysis was performed on DNA extracts from bacteria on LJ-slants as previously described $[15,16]$, with 10 minutes of denaturation prior to hybridization at $95^{\circ} \mathrm{C}$. Briefly, 2-3 $\mu$ l of each DNA sample was treated with RNAse prior to analysis. MLPA-probes hybridization was done overnight ( \pm $16 \mathrm{hrs}$ ) at $60^{\circ} \mathrm{C}$, followed by ligation at $54^{\circ} \mathrm{C}$ for 15 
minutes and then the ligase was inactivated at $98^{\circ} \mathrm{C}$ for 5 minutes. Subsequently, aliquots of the ligated products were amplified by PCR (35 cycles of 30" $95^{\circ} \mathrm{C}, 30^{\prime \prime} 60^{\circ} \mathrm{C}, 60^{\prime \prime} 72^{\circ} \mathrm{C}$, followed by a final $20^{\prime}$ $72^{\circ} \mathrm{C}$ ) with only one primer-pair, since all probes carry the same primer-sequence.

The amplified products were first analyzed on a $2 \%$ agarose gel and then by capillary electrophoresis. DNA from confirmed M. tuberculosis and M. bovis patients was used as positive controls and distilled water was used as a negative control. The phenotypic consequences of the presence of the various MLPA products are explained in Table 3.

Quality control

For quality control, DNA from 20 positive cultures collected from biopsy were randomly selected and tested at the Royal Tropical Institute (KIT) in Amsterdam, using the Genotype MTC kit (Hain, Germany) according to the manufacturer's instructions (HAIN, Life science). All 20 DNA samples proved to be $M$. tuberculosis (data not shown).

\section{Data analysis}

Data analysis by the AHRI data management unit used Stata/SE; release 7.0. Cross-tabulation was done to compare the proportion of HIV positives among culture-positive TBLN cases with the proportion of HIV positives among culture-negative lymphadenopathy cases. Pearson Chi- square test was used as statistical tool.

\section{Results}

Culture results were available from 156 of the 171 patients as follows: 107(69\%) were culture positive; $49(31 \%)$ showed no growth; 12 were contaminated; and 3 were lost by accident. Histological examination showed granulomas in 99/107 (93\%) of culturepositive patients and in 24/49 (49\%) of culture- negative patients. Seven of the culture positives could not be maintained and PCR was done using DNA extracted from the remaining 100 positive cultures. Ten $(5.8 \%)$ of the 171 TBLN patients were HIV seropositive. Six of the $10 \mathrm{HIV}$ seropositive were culture positive. There were no statistically significant differences between the proportions of HIV seropositivity among culture-positive TBLN patients and among culture-negative TBLN suspects (Table 1).

Table 1. Comparison of HIV status and TB Culture.

\begin{tabular}{|l|l|l|l|}
\hline \multirow{2}{*}{ HIV } & \multicolumn{2}{|c|}{ Culture } & \multirow{2}{*}{ Sum } \\
\cline { 2 - 3 } & Positive (107) & Negative (49) & \\
\hline Positive (10) & 6 & 4 & 10 \\
\hline Negative (146) & 101 & 45 & 146 \\
\hline Sum & 107 & 49 & 156 \\
\hline
\end{tabular}

Pearson Chi-square $=0.4, \mathrm{P}=0.546, \mathrm{OR}=0.7(95 \% \mathrm{CI}, 0.2-2.5)$

Figure 1 shows a representative gel picture of the RD10 PCR results using DNA extracted from biopsy cultures, and control reference strains of $M$. tuberculosis and M. bovis. M. bovis was not detected in any of the 100 positive biopsy cultures from TBLN patients.

Initially, RD10 based PCR was done using DNA extracted from 14 FNAs. Twelve out of 14 (86\%) FNAs tested were positive for 308 bp bands and indicated M. tuberculosis. However, PCR analysis using DNA from FNAs was abandoned due to weak band intensity (result not shown). Seven (50\%) among 14 of the FNAs tested by PCR had positive biopsy cultures, and all of these seven positive biopsy cultures were positive for $308 \mathrm{bp}$ bands which could

Figure 1. Use of PCR-based RD10 assay to identify M. tuberculosis and M. bovis on template

DNA extracted from biopsy cultures of TBLN patients.
1
23
45
7
8
9
10
11
12

6

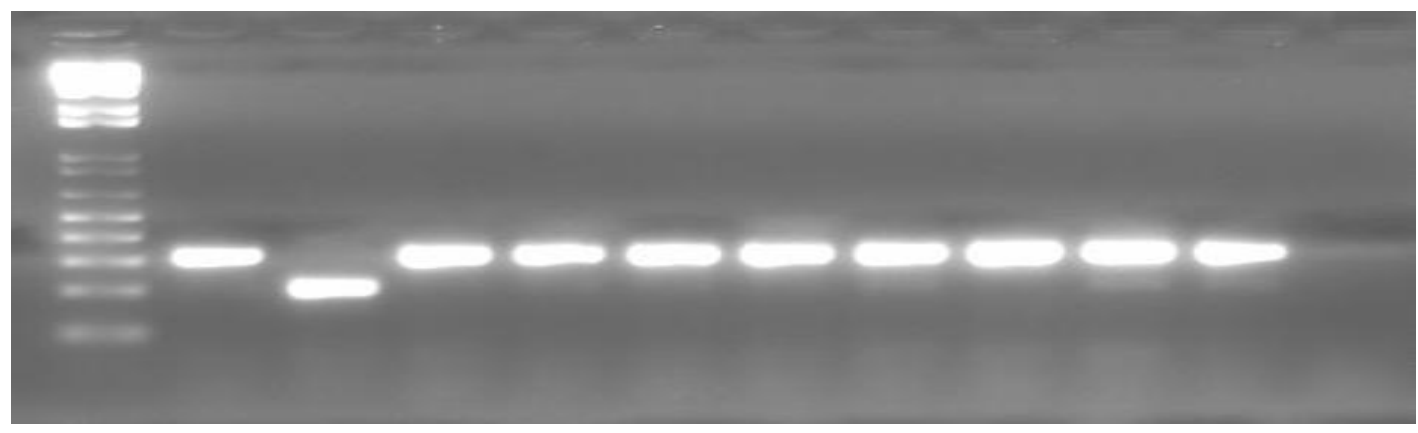

Lane 1= DNA molecular weight marker (100 bp - $1000 \mathrm{bp})$; Lane 2 = DNA from reference $M$. tuberculosis strain (H37RV); Lane 3= DNA from reference $M$. bovis strain (ATCC-19210); Lanes 4 through Lane 11 = DNA extracted from frozen bacteria of biopsy cultures from TBLN patients; Lane 12= negative control. 
Table 2. Summary of MLPA results

\begin{tabular}{|c|c|c|c|c|c|c|c|c|c|c|c|c|c|c|c|c|c|c|}
\hline $\begin{array}{l}\text { ठ } \\
\text { p. } \\
\stackrel{0}{0} \\
\text { है }\end{array}$ & 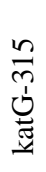 & $\begin{array}{l}\frac{n}{3} \\
\frac{\pi}{\Xi} \\
.\end{array}$ & 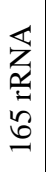 & $\begin{array}{l}\stackrel{0}{2} \\
\stackrel{1}{2} \\
\stackrel{0}{0}\end{array}$ & $\begin{array}{l}\vec{n} \\
\tilde{n} \\
\tilde{o} \\
\stackrel{0}{0}\end{array}$ & 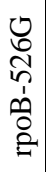 & 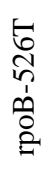 & $\begin{array}{l}\text { ते } \\
\tilde{n} \\
\stackrel{0}{0} \\
\stackrel{2}{2}\end{array}$ & 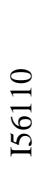 & $\begin{array}{l}\hat{\delta} \\
+ \\
\vdots \\
0 \\
\tilde{v} \\
\end{array}$ & 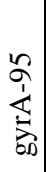 & 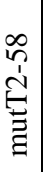 & $\begin{array}{l}\frac{1}{1} \\
\frac{1}{00}\end{array}$ & $\begin{array}{l}n \\
\frac{1}{00} \\
0\end{array}$ & $\begin{array}{l}\hat{n} \\
\vdots \\
0 \\
0\end{array}$ & है & Characteristics & $\begin{array}{l}\text { \#Strains With } \\
\text { profile }\end{array}$ \\
\hline $\mathbf{\square}$ & & & $\mathbf{\square}$ & & & & & & - & $\mathbf{a}$ & $\mathbf{\square}$ & & & $\square$ & & & PGG2- (Haarlem) & 2 \\
\hline $\mathbf{\square}$ & & & - & & & & & & - & $\mathbf{a}$ & $\mathbf{\square}$ & & & $\mathbf{\square}$ & & & PGG2- Haarlem & 29 \\
\hline $\mathbf{\square}$ & - & & - & & & & & & - & $\mathbf{a}$ & $\mathbf{\square}$ & & & - & & & PGG2- Haarlem, INH-resistant (via katG-S315T & 4 \\
\hline $\mathbf{\square}$ & - & & - & & & & & & - & - & - & & & & & & PGG2, INH-resistant (via katG-S315T) & 7 \\
\hline - & & & - & & & & & & - & - & & & & & & & PGG 3 & 10 \\
\hline - & & & - & & & & & & 口 & & - & & & & & - & M. bovis profile (pos. control) & \\
\hline
\end{tabular}

- : resulted in MLPA- product (SNP present) $\square$ : possibly resulted in MLPA- product (result inconclusive) PGG: principal Cenotypic Group
MLPA- probes are depicted in the columns on the left (vertical) and indicated the targeted regions or colons (16). In the columns "characteristics", the interpretation of the MLPA results is indicated and the last column, the number of strains resulted in specific MLPA profiles hs indicated. be $M$. tuberculosis. Six of the FNAs tested were culture negative and one FNA had no biopsy culture.

PCR-results showed that none of the patients' samples was infected with $M$. bovis. The same was true when DNA from cultured bacteria were analyzed by MLPA (Table 2). MLPA provided additional information on the strains, such as drug resistance to isoniazid ("katG-315", "inhA-15" mutations) and also principal genotype group (PGG). The $M$. bovis control was PGG1 with the TbD1region. The majority of the isolates $83 / 95(87 \%)$ belonged to PGG 2, and $35(42 \%)$ of those belonged to the Haarlem genotype. Merely 2/95 (2\%) strains were part of PGG 1 and 10/95 (11\%) of PGG 3. INH resistance was detected in 11/95 (12\%) of the strains, all of which were PGG 2 and were resistant via katGS315T mutations. None of the isolates carried the TbD1 fragment. This finding excludes all other members of the MTC including $M$. canettii and $M$. africanum. Moreover, our PCR identified RD10 sequences in all the isolates tested, further evidence that all strains isolated were $M$. tuberculosis.

\section{Discussion}

This study has shown that in culture-confirmed TBLN patients, $M$. tuberculosis was the only etiological agent identified when tested by PCR, using RD10 primers system and MLPA assay. The presence of granulomas in 24 of 49 culture-negative patients might be due to infection by either tuberculous or nontuberculous mycobacteria (NTM) which were not adequately detected by the sample culture. Our results are compatible with a study conducted in Butajira, Southern Ethiopia, which reported that in 23 patients identified with nontuberculous lymphadenitis by cytology, PCR confirmed the presence of MTC in 15 and in the remaining 8, MTC was not detected [21]. Moreover, a recent study conducted at AHRI using FNAs and biopsies of 117 TBLN patients from eastern, central and northern Ethiopia identified only M. tuberculosis as the etiological agent of TBLN by using the RD10 primers system [22]. In addition, MLPA results from our study clearly indicate that TbD1 was absent from all the isolates tested, confirming that all isolates are modern M. tuberculosis strains [23,24]. Collectively, these results suggest that $M$. bovis appears to be a rare etiological agent of TBLN in Ethiopia.

Our results contrast with previous studies that suggest $M$. bovis may be responsible for a larger proportion of TBLN cases. Kidane et al. in 2002 [5] reported PCR-based detection of $M$. bovis in $17 \%$ of lymph node FNAs obtained from patients diagnosed as TBLN by cytology. However, studies on the potential of nucleic acid amplification techniques (NAAT) to diagnose TBLN from clinical samples reported that these techniques produce variable and inconsistent results. NAATs should be applied in conjunction with conventional methods and interpreted in the context of clinical suspicion [14]. A 
Tanzanian study [8] on patients with different clinical manifestation of TB found that $16 \%$ were caused by M. bovis, $70.5 \%$ by $M$. tuberculosis, and $13.6 \%$ by other mycobacterial species. Moreover, the same study indicated that $26.8 \%$ of EPTB cases and only $4.3 \%$ of PTB cases were due to M. bovis. However, these results were based on biochemical typing which is less reliable and requires further confirmation with more sensitive and specific techniques, such as PCR.

Table 3. The MLPA-probes used in this study.

\begin{tabular}{|l|l|}
\hline \multicolumn{1}{|c|}{ probe } & \multicolumn{1}{c|}{ target/info } \\
\hline embB-306 & EMB resistance marker \\
\hline katG-315 & INH resistance marker \\
\hline inhA-15 & INH resistance marker \\
\hline 16S rRNA & 16S ribosomal RNA gene, MTB-complex specific \\
\hline rpoB-176 & RIF resistance marker \\
\hline rpoB-531 & RIF resistance marker \\
\hline rpoB-526G & RIF resistance marker \\
\hline rpoB-526T & RIF resistance marker \\
\hline rpoB-522 & RIF resistance marker \\
\hline IS6110 & insertion element IS6110, MTB-complex specific \\
\hline katG-463 & genotype marker, specific for PGG 2 and 3 \\
\hline gyrA-95 & genotype marker, specific for PGG 1 and 2 \\
\hline mutT2-58 & genotype marker, specific for Beijing 2 \\
\hline ogt-12 & genotype marker, specific for Beijing 2 \\
\hline ogt-15 & genotype marker, specific for Haarlem \\
\hline ogt-37 & genotype marker, specific for Beijing 3 and 4 \\
\hline TbD1 & absent in modern MTB strains \\
\hline
\end{tabular}

Probes were named after the gene and specific codon/region they target. A short summary of the information provided by the probes is given (15). EMB: ethambutol, INH: isoniazid, RIF: rifampicin, PGG: principal genotypic group.

Thus, using proper methods for detection is essential for conclusively identifying $M$. tuberculosis.

Differences between our results and those of Kidane et al. may also relate to the proportion of HIV-positive individuals in each study. HIV seropositivity was higher (27\%) in Kidane's study than in ours $(5.6 \%)$. Given that different regions of Ethiopia have different seroprevalences [25], it is possible that referral bias may account for these differences. This study's seroprevalence values more closely reflect overall seroprevalence in the Butajra region during the past decade [26]. Given other reported associations of HIV with M.bovis and $M$. tuberculosis [27,28], it is possible that HIV-infected individuals may be more prone to $M$. bovis infection and/or transmission. However, in our study, there were only six HIV seropositives among the 100 culture-positive TBLN cases and we could not see the association of HIV with M. bovis infection.

This study was limited by our utilization of solid culture media, which may have been suboptimal for the growth of $M$. bovis [29,30].

However, we further analyzed $M$. tuberculosis isolates by MLPA to define genotypes and drug sensitivity profiles and the results were consistent with those found in strains causing pulmonary tuberculosis in southern Ethiopia [31]. The majority of the isolates, 83/95 (87\%), belonged to PGG 2, and $35(42 \%)$ of those belonged to the Haarlem genotype, a virulent strain that is highly prevalent in Western Europe, Brazil, and Sub-Saharan Africa [32]. INHresistance was detected in 11/95 (12\%) of the strains. However, the current MLPA assay allows identification of only the most prevalent drugresistant mutations, so that this percentage may underestimate the total INH resistance. Nonetheless, the results obtained have revealed new information on drug resistance and genotype of TBLN isolates, prompting further research of these issues. The data presented in this study provide valuable epidemiological information.

Studies from the UK have shown that pasteurization of milk and an intensive animal TB control programme contributed to considerable reduction of bovine $\mathrm{TB}$ in both humans and cattle [33]. However, in developing countries there are no effective animal TB control programmes and surveillance, and the epidemiological and public health aspects of infection due to bovine TB are unknown [34]. Our data offer no evidence for transmission of the etiological agent of TBLN from cattle to human and we therefore suspect TBLN is transmitted from human to human. The absence of $M$. bovis as an etiological agent of TBLN in patients from Butajira and other parts of Ethiopia suggest that bovine TB is rare in humans. Further studies on the epidemiology, including the relationship with HIV infection, the manifestation of TBLN, and its association with certain $M$. tuberculosis lineages, such as Haarlem genotype, are warranted.

\section{Acknowledgements}

The authors acknowledge AHRI core funding from NORAD and Sida/SAREC for the study. Miss Meseret Habtamu is acknowledged for doing the cultures. Mrs. Banchayehu Gualu and Mr. Kiros Ayenew are acknowledged for their contributions in processing the 
formalin fixed biopsies and making them ready for histology examination. We are very thankful to Dr. Solomon Goshu and Dr. Berhanu Nega at the Butagira and Martha NGO Hospitals respectively for their contribution in doing the minor surgery to take the lymph-node biopsies. Mrs. Hiwot Tilahun and the data entry clerks at the data management unit at AHRI are highly acknowledged for their contribution in the data entry, cleaning, and assistance in the analysis. Our in-depth appreciation goes to Mr. Bamelaku Tilahun who faithfully transported the samples from the study site to AHRI.

\section{References}

1. World Health Organization (2008) Global Tuberculosi Control: Surveillance, planning, financing. Geneva, World Health Organization, (WHO/HTM/TB/2008.393).

2. Ministry of Health of Ethiopia (2005/2006) National Tuberculosis and Leprosy Control Program (NTLC), Ministry of Health of Ethiopia.

3. Cleaveland S, Shaw DJ, Mfinanga SG, Gabriel S, Rudovick RK, Ernest E and Michael S (2007) Mycobacterium bovis in rural Tanzania: Risk factors for infection in human and cattle population. Tuberculosis Edinburgh. Scotland 87: 3043.

4. Grange J M. (2001) Mycobacterium bovis infection in human beings. Tuberculosis 81: 71-77.

5. Kidane D, Olebo JO, Habte A, Negesse Y, Aseffa A, Abate G, Yassin A, Bereda K and Harboe M (2002) Identification of the causative organism oftuberculous Lymphadenitis in Ethiopia by PCR. J Clin Microbiol 40: 4230-4234.

6. Prasad H K, Singhal A, Mishra A, Shah NA, Katock VM, Thakral SS, Singh DV, Chumber S, Bal S, Aggarwal S, Padma MV, Kumar S, Singh MK, Acharya SK (2005) Bovine tuberculosis in India: Potential basis for zoonosis. Tuberculosis 85: 421-428.

7. Ameni G, Aseffa A, Engers H, Young D, Gordon S, Hewinson G, Vordiermier M (2007) Both prevalence and severity of pathology of bovine tuberculosis are higher in Holstein than in Zebu breeds under cattle field husbandry in Central Ethiopia. Clin Vaccine Immunol 14: 1356-1361.

8. Kazwala RR, Daborn CJ, Sharp JM, Ambarage DMK, Jiwa SFH, Mbembati N (2001) Isolation of Mycobacterium bovis from human cases of cervical adenitis in Tanzania: a cause for concern? Int J Tubec Lung Dis 5: 87-88.

9. Oloya J, Opuda-Asibo J, Kazwala R, Demelash AB, Skjerve E, Lund A, Johansen TB, Djonne B (2007) Mycobacteria causing human cervical Lymphadenitis in pastoral communities in the Karamajo region of Uganda. Epidemiol Infect June 29: 1-8.

10. Pereenboom RM., Richter C, Swai AB, Kitnya J, Mtoni L, Chande H, Kazema RR, Mwakyusa DH, Maselle SY (1994) Diagnosis of tuberculous lymphadenitis in an area of HIV infection and limited diagnostic facilities. Trop Geogr Med 46: 288-289.

11. Omani K, Kazi BM, Ahmed K, Karamat KA (2007) Frequency of tuberculous lymphadenitis detection at a single laboratory in Islamabad. J Coll Physicians Surg Pak 17: 410-412.

12. Parsons LM, Brosch R, Cole ST, Somoskövi A, Loder A, Bretzel G, Van-Soolingen D, Hale YM, Salfinger M (2002) Rapid and simple approach for identification of Mycobacterium tuberculosis complex isolates by PCR based genomic deletion analysis. J Clin Microbiol 40: 23392345.

13. Portillo P Del, Thomas M C, Martinez E, Maravon C, Valladares B, Patarroyo EM, Lopez MC (1996) Multiprimer PCR system for differential identification of Mycobacteria in clinical samples. J Clin Microbiol 34: 324-328.

14. Daley P, Thomas S, Pai M (2007) Nucleic acid amplification tests for the diagnosis of tuberculous lymphadenitis: A systematic review. Int J Tuberc Lung Dis 11: 1166-1176.

15. Schouten JP, McElqunn CJ, Waaijer R, Zwijnenburg D, Diepvens F, Pals G (2002) Relative quantification of 40 nucleic acid sequences by multiplex ligation dependent probe amplification. Nucleic Acids Res 30: e57.

16. Bergval IL, Vijzelaar RNCP, Dalla CostaER, Schuitema ARJ, Oskam L, Kritski AL, Klatser PR and Anthony RM (2008) Development of Multiplex Assay for Rapid Characterization of Mycobacterium tuberculosis. J Clin Microbiol 46: 689-699.

17. http://www.csa.gov.et/text_files/zena_Masa_Oct_1999.pdf].

18. Appendices (2002) Manual of the National Tuberculosis and Leprosy Control (NTLC). Ministry of Health of Ethiopia.

19. Talbot EA, Williams DL, Frothing-ham R (1997) PCR identification of Mycobacterium bovis BCG. J Clin Microbiol 35: 566-569.

20. van Soolingen D, Hermans PEM, de Haas PEW, van Embden JDA (1995) RFLP analysis of Mycobacteria: Manual for fingerprinting of $M$. tuberculosis Strains. National Institute of Public Health and Environmental Protection, Bilthoven, The Netherlands.

21. Yassin MA, Olobo JO, Kidane D, Negesse Y, Shimeles E, Taddesse A,Demissie A, Britton S, Harboe M, Aseffa A, Abate G (2003) Diagnosis of tuberculous lymphadenitis in Butajira, rural Ethiopia. J Infect Dis 35: 240-244.

22. Rahel I, Jan van den H, Yimtubezinash W, Mulat A, Cherinet G, Yared N, Teklu B, Senait A, Berhanu S, Kibrebeal M, Lawrence Y, Hiwot T, Zerihun T, Abraham A (2009) Is tuberculous lymphadenitis over-diagnosed in Ethiopia? Comparative performance of diagnostic tests for mycobacterial lymphadenitis in a high-burden country. Scandinavian Journal of Infectious Diseases Apr 20: 1-7.

23. R.Brosch, S.V.Gordon, M.Marmiesse, P. Brodin, C.Buchrieser, K.Eiglmeier, T.Garnier, C.Gutierriez, G.Hewinson, K.Kremer, L.M. Parsons, A.S.Pym, S.Samper, D.van.Soolingon and S.T.Cole (2002) A new evolutionary scenario for the Mycobacterium tuberculosi s complex. PNAS 99: 3684-3689.

24. Helen D Donoghue, Mark Spigelman, Charles L Greenblatt, Galit Lev- Maor, Gila Kahila Bar-Gal, Carney Matheson, Kim Vernon, Andreas G Nerlich, and Albert R Zink (2004) Tuberculosis: from prehistory to Robert Koch, as revealed by ancient DNA. Lancent Infectious Diseases 4: 584-592.

25. Abebe Y, Schaap A, Mamo G, Negussie A, Darimo B, Wolday D, Sanders EJ (2003) HIV prevalence in 72, 000 urban and rural male army recruits, Ethiopia, 1999-2000. Ethiopian Med J 41: 25-30.

26. Thoen CO, Steele JH (1995) Mycobacterium bovis infection in animals and humans. Iowa State University Press, Ames: 3-68.

27. Grange JM, Daborn C, Cosivi O (1994) HIV related tuberculosis due to Mycobacterium bovis. Eur Respir J 7: 1564-1566.

28. Ramos A, Noblejas A, Martin T, Varela A, and Daza R, Samper S (2007) Prolonged survival of an HIV infected 
patients with multi-drug Mycobacterium bovis infection treated with surgical resection. Clin Infect Dis 39: e53-e55.

29. Collins $\mathrm{CH}$, Grange JM: The bovine tuberculle bacillus (1983) J Appl Bacteriol 55: 13-29.

30. Thoen CO, Steele JH (1995) Mycobacterium bovis infection in animals and humans. Iowa State University Press, Ames: 3-68.

31. Abate G (2002) Drug-resistant tuberculosis in Ethiopia: problem scenarios and recommendation. Ethiop Med J 40: 79-86.

32. Laurent XN, Kassa-Kelembho E, Vultos TD, German Z, Jean R, Carmen L, Carlos M, Jesus B, Antoine T and Brigitte G (2006) Multidrug-resistant Mycobacterium tuberculosis, Bangui, Central African Republic. Emerg Infect Dis 12: 1454-1456.

33. Grange J M, Macolm Yates D (1994) Zoonotic aspects of Mycobacterium bovis Infection. Vet Microbiol 40: 1012115.

34. Cosivi O, Grange JM, Daborn CJ, Ravigline MC, Fugikura T, Cousins D, Robinson RA, Huchzermeyer HFAK, Kantor
Ide, Meslin FX (1998) Zoonotic Tuberculosis due to Mycobacterium bovis in Developing Countries. Emerg Infect Dis 4:5-70.

\section{Corresponding Author}

Demissew Beyene

Armauer Hansen Research Institute (AHRI)

ALERT Campus, Jimma Road, Addis Ababa, Ethiopia

PO Box 1005

Fax: 251-1-3-211563

Tel no: 251-1-3-211334

Email: beyene88@gmail.com

Conflict of interest

No conflict of interest is declared. 